\title{
Superior mesenteric arteriovenous fistula: Case report and literature review
}

\author{
JOSEPH C. BENEDETTO, DO \\ RICHARD F. LISZEWSKI, DO, FACOS
}

\begin{abstract}
A case of postoperative superior mesenteric arteriovenous (AV) fistula is presented. Review of the literature reveals the lesion to be an uncommon complication of abdominal trauma and surgery; less than 35 cases have been reported since the first in 1960 . The fistula should be suspected in any patient who has undergone abdominal surgery or who has recently sustained penetrating abdominal trauma and who presents with crampy abdominal pain, diarrhea, and an abdominal bruit. Identification and precise localization is best accomplished by selective superior mesenteric arteriography. Symptoms and signs are those of transient intestinal ischemia and portal hypertension in association with an abdominal bruit or thrill. However, evidence of a high cardiac output state also may be present. In the reported case, the patient related episodes of chest tightness and a syncopal event. Mortality is due primarily to the sequelae of portal hypertension, which result from increased systemic-to-portal inflow of blood through the fistula. Therefore, early correction should be undertaken. Treatment should consist of laparotomy with ligation and division of the fistula. Successful percutaneous transcatheter embolization of a superior mesenteric AV fistula has been reported.
\end{abstract}

Superior mesenteric arteriovenous (AV) fistula is an uncommon complication following penetrating or blunt abdominal trauma, surgery, or aneurysm. To date, fewer than 35 cases of superior mesenteric AV fistula have been documented since
Movitz and Finne's ${ }^{1}$ first report in 1960.

We present a case in which the fistula was secondary to right hemicolectomy for invasive carcinoid of the base of the appendix. The significance of superior mesenteric AV fistula, as well as its etiology, diagnosis, and treatment, is discussed.

\section{Report of case}

A 27-year-old man was admitted with a chief complaint of lower right quadrant abdominal pain. Six months earlier, the patient underwent right hemicolectomy for invasive carcinoid of the base of the appendix, which had been found incidentally at surgery for acute suppurative appendicitis.

Postoperatively, he had experienced recurrent episodes of lower right quadrant abdominal pain associated with nausea and loose bowel movements. Symptomatic treatment with analgesics had produced no pain relief. He also related episodes of chest tightness on exertion, which had been relieved by rest; these were worse at the end of the day. The patient denied emesis, melena, hematochezia, fever, chills, night sweats, or flushing associated with the pain. Results of a postoperative 5-hydroxyindoleacetic acid determination (24-hour urine collection) had been within normal limits.

Two weeks prior to admission, the patient had experienced abdominal pain and syncope. He was examined in the emergency department, treated with tylenol with codeine, and released. One week later, he was examined by his surgeon, who discovered an abdominal bruit in the lower right quadrant. Ultrasonography of the abdomen and aorta disclosed no abnormalities.

On admission, selective superior mesenteric arteriogaphy revealed a direct communication between an ileal branch of the superior mesenteric artery and vein, with rapid filling of the superior mesenteric vein and portal vein (Fig 1). Also, marked dilatation of the superior mesenteric vein was noted. At laparotomy, a thrill was palpated in the mesentery. Exploration confirmed the arteriographic report, with AV fistula found between an ileal branch of the superior mesenteric artery and vein. Following quadruple ligation of the fistula, the thrill 


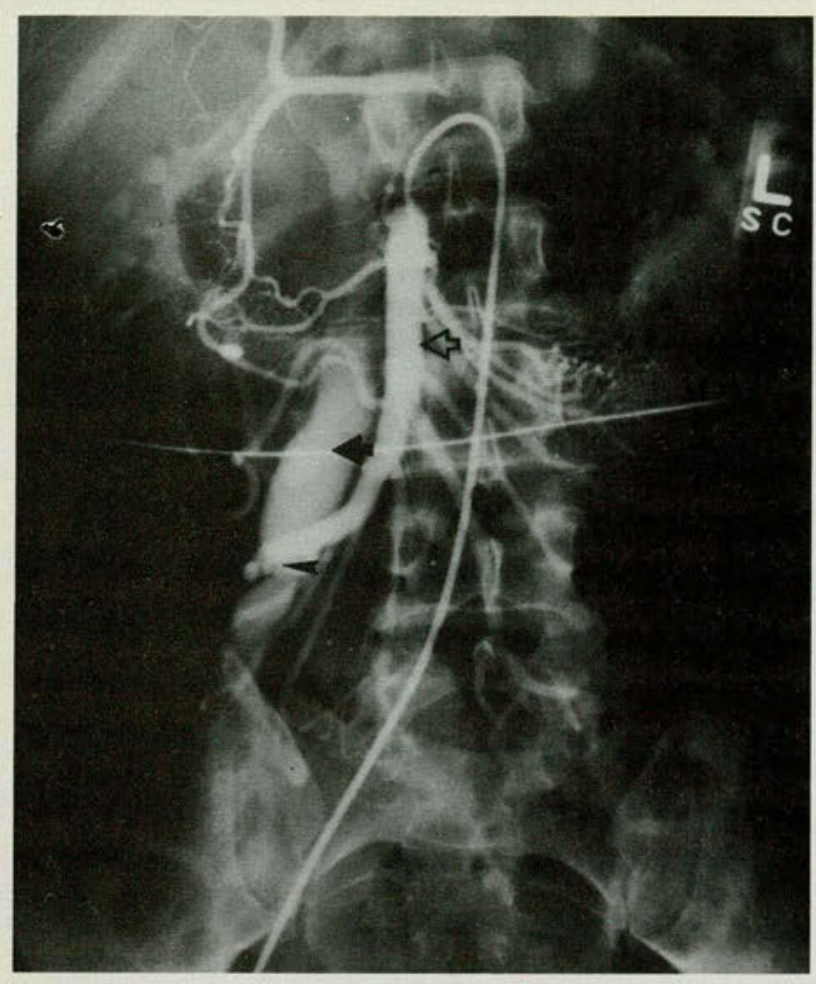

Fig 1 Selective superior mesenteric arteriogram shows early filling of superior mesenteric vein (closed arrow) and portal venous system. The open arrow indicates the superior mesenteric artery; the arrowhead indicates the AV fistula.

resolved.

The patient had an uncomplicated recovery, with resolution of the abdominal bruit, abdominal pain, diarrhea, and chest tightness. He was discharged on the sixth postoperative day.

\section{Discussion \\ Etiology}

Penetrating abdominal trauma has been the most commonly reported ${ }^{2-10}$ cause of superior mesenteric AV fistula. In decending order of incidence, gunshot, stab, and shotgun wounds and shrapnel injury have been reported causes. ${ }^{10}$ Injury usually has involved an artery and vein in close proximity. ${ }^{11}$

Surgery is the next most common etiology. The majority of postoperative superior mesenteric AV fistulas have occurred after bowel resection. However, the fistula has been reported ${ }^{12,13}$ after gastrectomy and laparotomy for lysis of adhesions without bowel resection. The direct cause of postsurgical fistula is the use of mass ligatures in the mesentery, which provides the initial arteriovenous communication required for the formation of a fis- tula. ${ }^{11-15}$ Moreover, Lee and associates ${ }^{12}$ have commented that mesenteric vessels are especially vulnerable to fistula formation "because they course closely together anatomically."

There have been isolated reports of superior mesenteric AV fistula resulting from blunt abdominal trauma ${ }^{10}$ and of spontaneous AV fistula formation secondary to rupture of a superior mesenteric artery aneurysm into its adjacent vein ${ }^{16}$. However, such causes are rare.

\section{Diagnosis}

The diagnosis of superior mesenteric AV fistula generally can be made on the basis of history and physical examination. Crampy abdominal pain, diarrhea, an abdominal bruit or thrill, and, occasionally, the presence of signs of portal hypertension, especially in patients with a history of bowel surgery or penetrating abdominal trauma, should raise the clinical suspicion of mesenteric AV fistula. ${ }^{5,11,13}$

Crampy abdominal pain and diarrhea have been attributed to transient ischemia of that portion of bowel distal to the fistula. ${ }^{3,5,9-11}$ Nicholas and Lane ${ }^{5}$ state that "the fistula can produce ischemia on both an arterial and a venous basis." Ischemic changes in the bowel, including discoloration and mucosal ulceration, also have been reported. ${ }^{5,12}$ Venous congestion with associated hemorrhage into the muscular layers of bowel wall and edema of bowel and mesentery have been reported ${ }^{1,5,6,12}$ at laporatomy and at autopsy. Reported cases ${ }^{6,9}$ of clinical presentation as abdominal pain, bloody diarrhea, fever, and leukocytosis without infection support this theory.

In Bole and coworkers ${ }^{2}$ case, hemodynamic studies demonstrated a high flow state across the AV fistula, with markedly diminished blood flow distally. These findings correlated with arteriographic findings of delayed visualization of the superior mesenteric artery distal to the shunt. They also support the theory of bowel ischemia as the cause of the clinical symptoms and signs of superior mesenteric AV fistula.

Hyperkinetic, turbulent blood flow across the fistula accounts for the presence of a bruit or thrill, ${ }^{2}$ which was present in virtually all reported cases, ${ }^{10}$ including ours. Hemodynamic alterations in patients with superior mesenteric AV fistula are seen primarily in the portal circulation. Portal hypertension and its sequelae secondary to abnormal systemic-to-portal blood flow have been documented. $3,5,6,8,10,12,13$ Death from superior mesenteric AV fistulas has resulted in approximately $10 \%$ of 
cases from the sequelae of portal hypertension. ${ }^{10}$

At first, it was thought ${ }^{3,6}$ that adverse systemic hemodynamic effects from systemic-to-portal fistulas were minimal or absent because of the damping effect created by the interposition of the liver between the abnormal AV communication and the right side of the heart. Unlike patients with systemic AV fistulas, there initially appeared to be no propensity by patients with systemic-to-portal fistula to develop clinical signs of a high cardiac output state. ${ }^{8}$ However, later reports ${ }^{2,5,13}$ have documented increased circulatory demand, which was reflected primarily as increased cardiac output. In a case of superior mesenteric AV fistula reported by Paloyan and associates, ${ }^{15}$ symptoms and signs of a high cardiac output state included cardiomegaly, hypertension, electrocardiographic demonstration of left ventricular hypertrophy, and peripheral edema. All cardiac abnormalities resolved after correction of the fistula. It is clear that while major hemodynamic effects occur in the portal circulation, there is potential for serious cardiac complications in patients with superior mesenteric AV fistula.

The single best diagnostic study is selective superior mesenteric arteriography, although diagnosis by digital subtraction angiography, ultrasonography, computed tomography, and magnetic resonance imaging has been reported. ${ }^{16,17}$ Arteriography allows precise definition and localization of the fistula, ${ }^{10-12}$ as well as evaluation of the portal venous system. ${ }^{12}$ Angiographic findings demonstrate early filling of the superior mesenteric vein and portal system, with dilatation of the mesenteric and portal venous system. Narrowing and delayed filling of the superior mesenteric artery distal to the fistula may also be observed.

Although aortography may also be a useful procedure, flush aortography may not demonstrate the fistula if it overlies an opacified aorta..$^{9-11}$ Consequently, if aortography alone is used, biplanar aortography should be the procedure of choice. ${ }^{10,11}$

\section{Treatment}

Recommendations have been made regarding prevention of postsurgical superior mesenteric AV fistula since the first reported case by Movitz and Finne, ${ }^{1}$ who stressed control with individual ligation of contiguous arteries and veins and avoidance of mass ligatures or transfixion ligatures. Subsequent authors ${ }^{11,12,14}$ have echoed their recommendations.

Prevention of post-traumatic superior AV fistula is less feasible, however, and early recognition and treatment should be the rule. At least one case report ${ }^{7}$ described recognition and treatment at primary laparotomy of a superior mesenteric AV fistula secondary to stab wound.

Surgical treatment generally consists of ligation and division of the fistula. However, depending on the fistula's size and location and associated pathologic findings, treatment might require arterial or venous reconstruction or bowel resection. ${ }^{12,14}$

In 1982, Uflacker and Saadi ${ }^{18}$ described the successful use of transcatheter embolization of a superior mesenteric AV fistula with stainless steel spring coils. While percutaneous transcatheter embolization is an attractive alternative to surgery, its use for fistulas of the mesenteric circulation is too limited to draw conclusions about its place in the treatment of these lesions. However, with advances in technology and expertise in interventional radiology, the procedure holds promise for the future.

\section{Comment}

The major significance of superior mesenteric AV fistula is its adverse effects on the portal circulation and blood supply to the bowel distal to the fistula. Mortality from superior mesenteric AV fistula can be directly attributed to these effects. ${ }^{6,10}$ Therefore, diagnosis should lead to immediate treatment.

It appears that size, location, and duration of the fistula determine the severity of its effect upon the systemic and portal circulatory systems, as well as upon the severity and nature of symptoms. ${ }^{5,11}$ Large, proximally located lesions tend to have the most pronounced effect on portal circulation, cardiac output, and blood supply to the bowel distal to the fistula, while smaller, more distal lesions have a lesser effect. ${ }^{11}$

Clinically apparent high cardiac output states with its sequelae, while rare, may be significant in these cases. ${ }^{15}$ Our patient had symptoms attributable to a high cardiac output state, including chest tightness and a syncopal event. However, adequate hemodynamic measurements were not made to document the cardiac output status.

\section{Summary}

A case of postoperative superior mesenteric arteriovenous fistula has been presented. Its etiology, diagnosis, and management are discussed. Regardless of its presentation, the potentially serious effects of superior mesenteric AV fistula have been documented, and the only prerequisite to treatment should be its diagnosis. 
1. Movitz D, Finne B: Postoperative arteriovenous aneurysm in mesentery after small bowel resection. JAMA 1960;173:42-44.

2. Bole P, Andronaco JT, Purdy R: Superior mesenteric arteriovenous fistula secondary to a gunshot wound: A case report and review of the literature. J Cardiovasc Surg 1973;14:456-459.

3. Forbes HW, Thompson CQ, Smith JW: Mesenteric arteriovenous fistula after a gunshot wound. J Trauma 1969;9:806-811.

4. Hunt TK, Leeds FH, Wanebo HJ, et al: Arteriovenous fistulas of major vessels in the abdomen. J Trauma 1971;11:483-493.

5. Nicholas GG, Lane MF: Traumatic superior mesenteric artery-superior mesenteric vein fistula. $J$ Trauma 1974;14:344-347.

6. Rabhan NB, Guillebeau JG, Brackney EL: Arteriovenous fistula of the superior mesenteric vessels after a gunshot wound. N Engl J Med 1962;266:603-605.

7. Smith CJ: Recognition and treatment of a mesenteric arterio-venous fistula at primary laparotomy for a loin stab wound. Med J Aust 1971;1:747-748.

8. Spellman MW, Mandal A, Freeman HP, et al: Successful repair of an arteriovenous fistula between the superior mesenteric vessels secondary to a gunshot wound. Ann Surg 1967;165:458-463.

9. Varner JE, Oliver RI: Superior mesenteric arteriovenous fistula. Ann Surg 1969;170:862-865.

10. Wood M, Nykamp PW: Traumatic arteriovenous fistula of the superior mesenteric vessels. J Trauma 1980;20:378-382.

11. Diehl JT, Beven EG: Arteriovenous fistulas of the mesenteric vessels: Report of a case and review of the literature. J Cardiovasc Surg 1982;23:334-337.
12. Lee KE, Kishore P, Hardin CA: Post-surgical arteriovenous fistula: Acquired AV fistula of the mesentery. J Kansas Med Soc 1974;75:87-90. 13. van Way CW, Crane JM, Riddell DH, et al: Arteriovenous fistula in the portal circulation. Surgery 1971;70:876-890.

14. Munnell ER, Mota CR, Thompson WB: Iatrogenic arteriovenous fistula: Report of a case involving the superior mesenteric vessels. Am Surg 1960;26:738-744

15. Paloyan D, Collins PA, Washburn FP: Superior mesenteric arteriovenous fistula. Am Surg; 1974;40:481-484.

16. Knox M, Chuang VP, Stewart MT: Superior mesenteric aneurysm and arteriovenous fistula: Angiographic and CT features. AJR $1974 ; 145: 383-384$

17. Amparo EG, Higgins CB, Hricak H: Primary diagnosis of abdominal arteriovenous fistula by MR imaging. J Comput Assist Tomogr $1984 ; 8: 1140-1142$.

18. Uflacker R, Saadi J: Transcatheter embolization of superior mesenteric arteriovenous fistula. AJR 1982;139:1212-1214.

From the Department of Surgery, University of Medicine and Dentistry-School of Osteopathic Medicine, Kennedy Memorial Hospitals-University Medical Center, Stratford, NJ.

Reprint requests to Dr Benedetto, UMDNJ-KMH, Stratford Division, 18 E Laurel Rd, Stratford, NJ 08084. 


\section{Introducing a Double Strength tablef to subdue pain/inflammation}



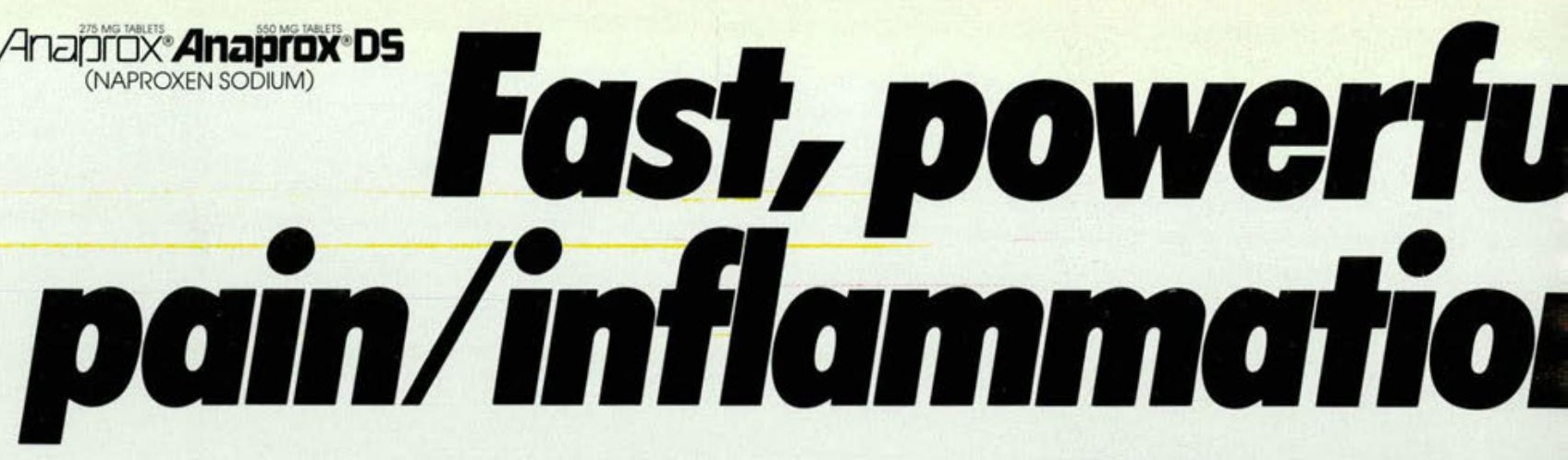

Now, there is ANAPROX Double Strength that gives you more analgesic/anti-inflammatory activity per dose. It joins the proven ANAPROX $275 \mathrm{mg}$ strength to provide you with greater dosage flexibility and convenience.

- Twice the strength-550 mg ANAPROX per tablet.

Dosage- The daily dosage of $1650 \mathrm{mg}$ can be used for limited periods when a higher level of analgesic/anti-inflammatory activity is required. Do not exceed this daily dose.

- Greater analgesic/anti-inflammatory activity in each tablet-reduces inflammation as it relieves the pain.

- No oral analgesic works faster-onset of pain relief may occur as fast as 20 minutes.

- Efficacy equal to the narcotic combinationseffective analgesia with a potent anti-inflammatory bonus the codeine combinations and propoxyphene napsylate can't offer.

- Non-narcotic-no narcotic-type side effects* or clinical limitations.

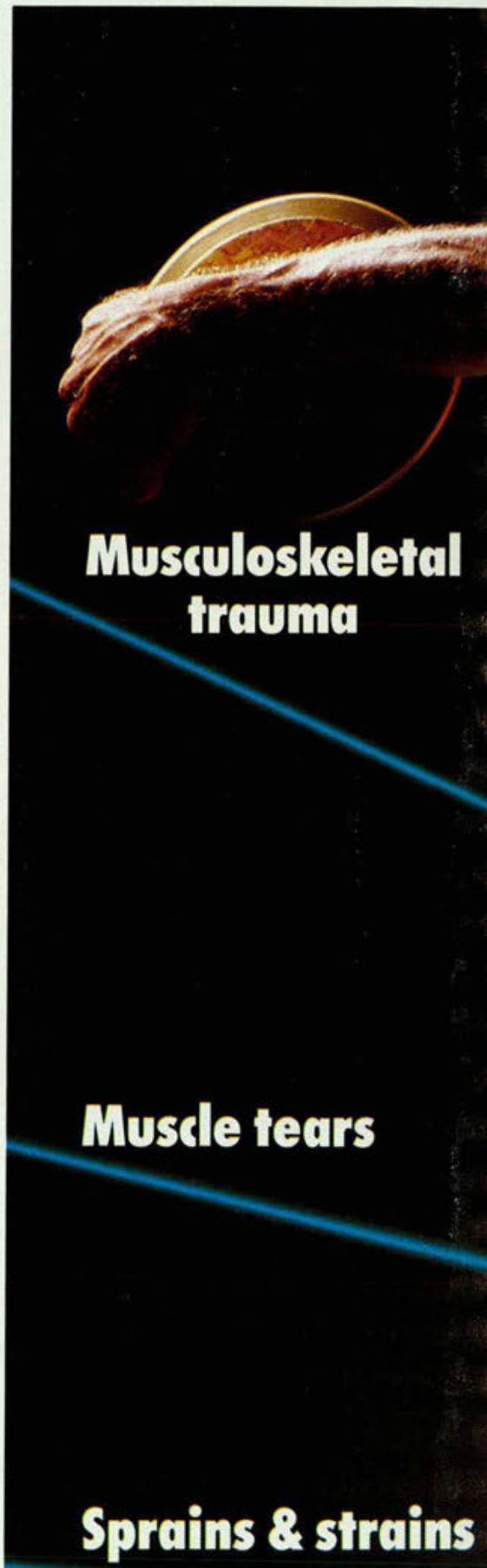


offer for the

OU Heal eVery doy

\section{Bursitis}

\section{Tendinitis}

Joint injury

Introducing 


\section{Fast, powerful relief for the pain inflammation you see every day}
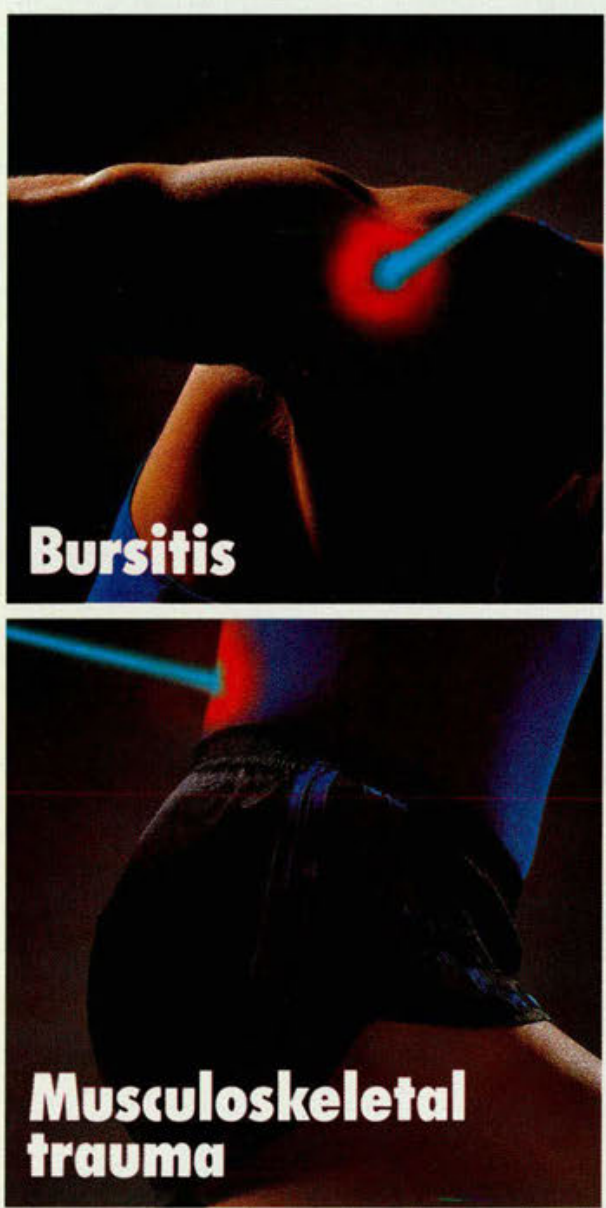

ANAPROX ${ }^{\circ}$ and ANAPROX DS (naproxen sodium) Tablets

Brief Summary:

Indications: Relief of mild to moderate pain; treatment of primary dysmenorrhea. Treatment of rheumatoid arthritis, osteoarthritis, juvenile arthritis, ankylosing spondylitis, tendinitis and bursitis, and acute gout. Contraindications: Patients who have had allergic reactions to NAPROSYN. ANAPROX or ANAPROX DS or in whom aspirin or other NSAIDs induce the syn. drome of asthma, rhinitis, and nasal polyps. Because anaphylactic reactions drome of asthma, minitis, and nasal polyps. Because anaphylactic reactions usually occur in patients with a history of such reactions, question patients for
asthma, nasal polyps, urticaria, and hypotension associated with NSAIDS asthma, nasal polyps, urticaria, and hypotension associated with NSAD Warnings: Peptic ulcers and GI bleeding have been reported in patients on NSAIDs, including naproxen sodium. In patients with GI bleeding or active peptic ulcer, start an anti-ulcer regimen, weigh benefits and risks of treatment, and monitor patient. Give to patients with history of GI disease only under close supervision and after reading Adverse Reactions section.

Precautions: DO NOT GIVE NAPROSYN" (NAPROXEN) CONCOMITANTIY WITH ANAPROX` OR ANAPROX` DS (NAPROXEN SODIUM) SINCE BOTH CIRCULATE IN PLASMA AS THE NAPROXEN ANION. Acute interstitial nephritis with hematu. ria proteinuria and nephrotic syndrome has been reported Patients with impaired renal function, heart failure, liver dysfunction, taking diuretics, and the elderly are at greater risk of overt renal decompensation. If this occurs, dis. continue the drug. Use with caution and monitor serum creatinine and/or creatinine clearance in patients with significantly impaired renal function. Use caution in patients with baseline creatinine clearance less than $20 \mathrm{ml} /$ minute. Use the lowest effective dose in the elderly or in patients with chronic alcoholic liver disease or cirrhosis. With NSAIDs, borderline elevations of liver tests may occur in up to $15 \%$ of patients. They may progress, remain unchanged, or be transient with continued therapy. Flevations of SCPT Or SCOT occurred in con transient with contined therapy Elevtions Of SCP. or SCOF occured in con including jaundice and fatal hepatitis, have been reported rarely. If liver disease develops or if systemic manifestations occur (e \&. eosinophilia or rash), discon. tinue therapy. If steroid dosage is reduced or eliminated during therapy, do so slowly and observe patients closely for adverse effects, including adrenal insufficiency and exacerbation of arthritis symptoms. Determine hemoglobin values periodically for patients with initial values of 10 grams or less who receive long. term therapy. Peripheral edema has been reported. For patients with restricted
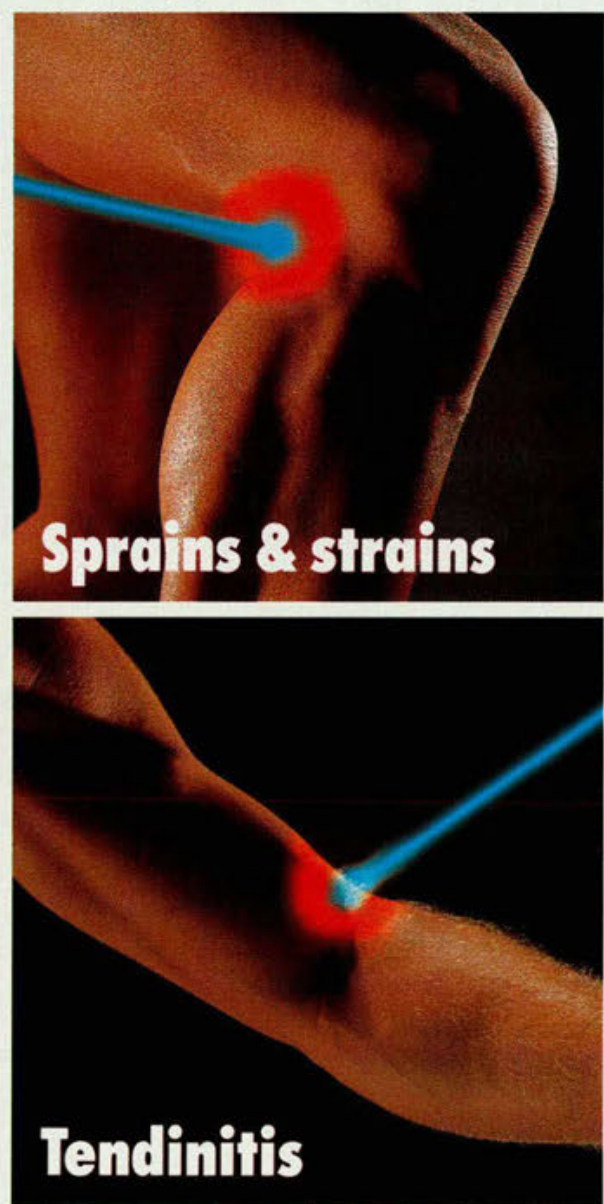

sodium intake, note that each tablet contains approximately $25 \mathrm{mg}$ or $50 \mathrm{mg}$ ( 1 or $2 \mathrm{mEq}$ ) sodium. Use with caution in patients with fluid retention, hyperten. sion or heart failure. The drug's antipyretic and anti-inflammatory activities may reduce fever and inflammation, diminishing their diagnostic value. Conduct ophthalmic studies if any change or disturbance in vision occurs. Information for Patients: Patients should use caution for activities requiring alertness if they experience drowsiness, dizziness, vertigo or depression during therapy.

Orug Interactions: Use caution when giving concomitantly with coumarin type anticaagulants: a hydantoin, sulfonamide or sulfonylurea: furosemide: lith. ium; beta-blockers; probenecid; or methotrerate.

Drug/aboratory Test Interactions: The drug may decrease platelet aggre. gation and prolong bleeding time or increase urinary values for 17 -ketogenic steroids. Temporarily stop therapy for 72 hours before doing adrenal function tests. The drug may interfere with urinary assays of 5 HIAA.

Carcinogenesis: A 2.year rat study showed no evidence of carcinogenicity. Pregnancy: Category B. Do not use during pregnancy unless clearly needed Avoid use during late pregnancy.

Nursing Mothers: Avoid use in nursing mothers.

Pediatric Use: Single doses of $25-5 \mathrm{mg} / \mathrm{kg}$ (as naproxen suspension), with total daily dose not exceeding $15 \mathrm{mg} / \mathrm{kg}$ /day, are safe in children over 2 years of age

Adverse Reactions: In a study. GI reactions were more frequent and severe in rheumatoid arthritis patients on $1500 \mathrm{mg} /$ day naproxen than in those on 750 $\mathrm{mg} /$ day (doses comparable to $1650 \mathrm{mg}$ and $825 \mathrm{mg}$ of naproxen sodium). In studies in children with juvenile arthritis, rash and prolonged bleeding times were more frequent, Gl and CNS reactions about the same, and other reactions less frequent than in adults. Incidence Greater Than 1\%. Probable Causal Relationship: Gl: The most frequent complaints related to the GI tract: constipation," heartburn," abdominal pain,* nausea," dyspepsia, diarrhea, stomatitis. CNS: headache," dizziness" drowsiness", light-headedness, vertizo. Dermatologic itching (pruritus)" skin eruptions." ecchymoses" sweating purpura Special Senses tinnitus** hearing disturbances, visual disturbances. Cardiovascular: edema*" dyspnea," palpitations. General: thirst. *Incidence of reported reaction $3 \%-9 \%$. Where unmarked, incidence less than $3 \%$. Incidence Less Than 1\%: Probable Causal Relationship: GI: abnormal liver function tests, colitis, GI bleeding and/or perforation, hematemesis, jaundice, melena, peptic ulceration with bleeding and/or perforation, vomiting Renal: glomerular
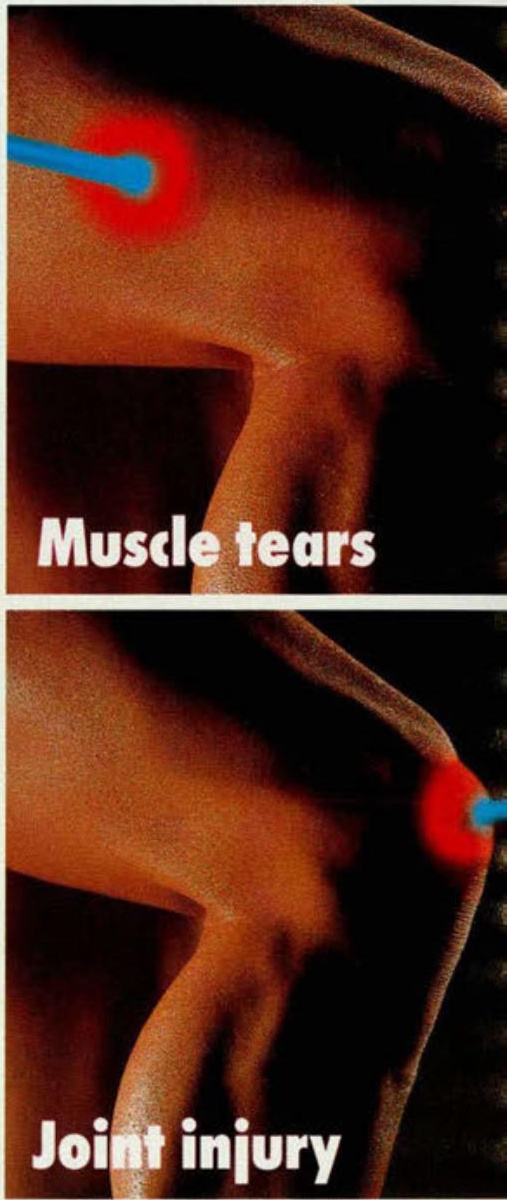

nephritis, hematuria, interstitial nephritis, nephrotic syndrome, renal dis Hematologic: agranulocytosis, eosinophilia, granulocytopenia, leukop thrombocytopenia. CNS: depression, dream abnormalities, inability to con trate, insomnia, malaise, myalgia and muscle weakness. Dermatologic: cia, photosensitive dermatitis, skin rashes. Special Senses: he impairment. Cardiovascular: congestive heart failure. Respiratory: eosino pneumonitis. General. anaphylactoid reactions, menstrual disorders, ph pneumonitis Gerl Causal Relationship Unknown: Hematologic: aplastic a chills and fever) Causal Relationship Unknown. Hematologic: aplastic an cognitive dystunction. Denmatologic epidermal lysis, ery thema multiforme, Stevens. John son syndrome, urticaria. Gl: ulce stomatitis. Cardiovascular: vasculitis. General: angioneurotic edema, glycemia, hypoglycemia.

Overdosage: May have drowsiness, heartburn, indigestion, nausea, vom Empty stomach and use usual supportive measures. Prompt administrat 5 grams activated charcoal may reduce drug absorption. Hemodialysis m appropriate in renal failure.

Dosage and Administration for Mild to Moderate Pain. Dysmenorrthe Acute Tendinitis and Bursitis: The recommended starting dose is $550 \mathrm{~m}$ lowed by $275 \mathrm{mg}$ every 6 to 8 hours, as required. The total daily dose shou

Dosage and Administration for Rheumatoid Arthritis, Osteoarthritis Ankylosing Spondylitis: The recommended dose in adults is $275 \mathrm{mg}$ or 55 twice daily. In patients who tolerate lower doses well, the dose may be incre to $1,650 \mathrm{mg}$ per day for limited periods when a higher level of inflammatorylanalgesic activity is required. At this dosage, physicians sh observe sufficient increased clinical benefits to offset potential increase Caution: Federal law prohibits dispensing without prescription. See package insert for full Prescribing Information. (c) Revised S SYNTEX

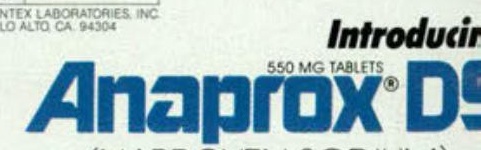

(NAPRÖXEN SODIUM) 